\title{
Microbiology
}

\section{COMMON PARASITES}

\section{Bobbi Pritt}

Division of Clinical Microbiology, Department of Laboratory

Medicine and Pathology, Mayo Clinic, Rochester, MN, USA

Humans can be infected with a diverse array of protozoa, helminths and arthropod ectoparasites. ${ }^{1}$ Many parasitic infections are found in tropical and subtropical settings and disproportionately affect individuals without access to adequate sanitary facilities and fresh water. ${ }^{2,3}$ Important examples of these infections include malaria, schistosomiasis, leishmaniasis, soiltransmitted helminths, food-borne trematodiases, filariasis, cysticercosis, taeniasis, echinococcosis, and myiasis. Many other parasitic infections, on the other hand, are found in both temperate and tropical climes and affect rich and poor alike. Examples of these include enterobiasis, head louse infestation, giardiasis, cryptosporidiosis, scabies, and trichomoniasis. Clinical microbiologists must be able to accurately identify the parasites that commonly infect the populations they serve, and know when to seek consultation for assistance in identifying uncommon parasites. They must also recognise frequently-encountered parasite mimics. This interactive, case-based presentation will cover a variety of common parasites and mimics, including those encountered in Australia and other resource-rich countries. Cases will include a discussion of laboratory testing approaches using traditional and molecular-based methods, and how to incorporate an algorithmic approach to parasite identification into routine practice.

\section{References}

1. Garcia LS, Arrowood M, Kokoskin E, et al. Laboratory diagnosis of parasites from the gastrointestinal tract. Clin Microbiol Rev 2017; 31: e00025-17.

2. World Health Organization. Neglected parasitic diseases. Cited 16 Oct 2019. https://www.who.int/neglected_diseases/en/.

3. World Health Organization. World Malaria Report 2018. Geneva: World Health Organization, 2018. https://www.who.int/malaria/ publications/world-malaria-report-2018/en/.

\section{SEROLOGY AND ANTIGEN TESTING: WHAT'S AVAILABLE AND WHAT'S USEFUL}

Harsha Sheorey

Department of Microbiology, St Vincent's Hospital, Melbourne, Australia

This talk will be presented as a part of the Parasitology Masterclass.

Numerous serological (antibody) tests and some antigen tests are available in Australian diagnostic laboratories. Some tests are available overseas. The availability of these tests in Australia or overseas will be presented. Also the specificity and sensitivity of various tests will be discussed and usefulness in various clinical scenarios will be presented along with limitations of some.

\section{MEDICAL ENTOMOLOGY FOR MICROBIOLOGY LABORATORIES}

Stephen Doggett

Department of Medical Entomology, NSW Health Pathology, Westmead Hospital, Westmead, NSW, Australia

Our Department is the main reference laboratory for the identification of medically important arthropods. Over the years 1988-2017, a total of 5,655 specimens were received. The Australian paralysis tick (Ixodes holocyclus) was the most common (708 submissions), followed by bird mites Ornithonyssus bursa (506), bed bugs Cimex spp. (149), moth flies Clogmia spp. (135), head lice Pediculus capitis (105), public lice Pthirus pubis (91), and scabies Sarcoptes scabiei (57). In 1,211 specimens, there was no evidence of any arthropods. Certain arthropods showed seasonal trends. Ixodes holocyclus larvae were most common in autumn, nymphs in winter, and adults in spring. Ornithonyssus bursa were more common through the spring to summer months. In contrast, Sarcoptes scabiei peaked during winter. Generally, there were few statistical differences between specimens submitted by females and males, although males were more associated with $P$. pubis, females with $P$. capitis, $O$. bursa, and specimens with no evidence for arthropods. Children under 10 were more likely associated with $I$. holocyclus and P. capitis, middle age people with Cimex, and the elderly with $S$. scabiei. Perhaps the most extraordinary trend is the annual growth in specimens that have no evidence for arthropods, with no obvious explanation for this trend.

\section{ESOTERIC CASE-BASED PRESENTATION}

\section{Bobbi Pritt}

Division of Clinical Microbiology, Department of Laboratory Medicine and Pathology, Mayo Clinic, Rochester, MN, USA

Parasites are all around us, infecting all types of living things and existing as both commensals and pathogens. Human parasites have co-evolved with us over the millennia and are generally well-known to clinical microbiologists: Plasmodium falciparum, $P$. ovale, $P$. vivax and P. malariae; Pediculus humanus capitis and $P$. h. humanus; Taenia solium and T. saginata, Cyclospora cayetanensis, Pentatrichomonas hominis, Pthirus pubis; Entamoeba histolytica; Trichomonas vaginalis; and others. ${ }^{1,2,3}$ Clinical microbiologists are also familiar with many of the zoonotic parasites that commonly infect humans in certain regions of the world: Diphyllobothrium and Dipbothriocephalus; Babesia species; Echinococcus granulosus and E. multilocularis; Trypanosoma cruzi; Plasmodium knowlesi; Toxoplasma gondii; Trypanosoma brucei rhodesciense; among others. Beyond these common human and zoonotic parasites are a seemingly endless number of parasites which only rarely infect humans, and in doing so, may result in substantial morbidity and 